\title{
Strategies for the management of OHSS: Results from freezing-all cycles
}

\author{
Edson Borges Jr. ${ }^{1,2}$, Daniela Paes Almeida Ferreira Braga ${ }^{1,2,3}$, Amanda S Setti ${ }^{1,2}$, Livia S. Vingris ${ }^{1}$, Rita Cássia S. \\ Figueira ${ }^{1}$, Assumpto Iaconelli Jr. ${ }^{1}$ \\ ${ }^{1}$ Fertility - Medical Group - Sao Paulo - Brazil \\ ¿Sapientiae Institute - Centro de Estudos e Pesquisa em Reprodução Assistida \\ ${ }^{3}$ Disciplina de Urologia, Área de Reprodução Humana, Departamento de Cirurgia, Universidade Federal de São Paulo
}

\begin{abstract}
Objective: To compare the use of $\mathrm{GnRH}$ agonist ( $\mathrm{GnRHa}$ ) or hCG trigger in potential OHSS patients undergoing freeze-all programs. We also compared the clinical outcomes when fresh versus freeze-thawed embryo transfers were performed in cycles with a high number of retrieved oocytes.

Methods: The study included potential OHSS patients who received GnRHa $(n=74)$ or hCG $(n=49)$ trigger. The protocols were compared with respect to the clinical outcomes. We also compared the clinical outcomes of cycles in which hCG trigger was used and more than 20 MII oocytes were retrieved when: fresh embryo transfer protocol $(n=153)$ or freeze-all protocol $(n=123)$ were performed.

Results: A decreased serum estradiol level, a decreased number of retrieved oocytes, an increased MII retrieved rate, and decreased fertilization rate was observed in the hCG when compared with the GnRHa group. No significant differences were noted concerning clinical outcomes. When fresh cycles were compared with frozen-thawed cycles, the estradiol serum level and the number of cryopreserved embryos were higher in the frozen-thawed cycles. The clinical pregnancy rate was higher among freeze-all cycles, as well as the implantation and cumulative pregnancy rates, when compared with fresh embryo transfer cycles.

Conclusion: The use of GnRHa trigger may be a good alternative to prevent the OHSS in patients presenting an extreme ovarian response to COS, leading to similar clinical outcomes, when compared with the traditional hCG trigger. Moreover, our findings demonstrated that the strategy of freezing-all embryos not only decreases the risk of OHSS but also leads to a better pregnancy rate.
\end{abstract}

Keywords: OHSS, Freezing, Thawing, hCG, GnRHa, Trigger

\section{INTRODUCTION}

Ovarian hyperstimulation syndrome (OHSS) is the dreadful complication of the controlled ovarian stimulation (COS), in which pharmacological doses of gonadotropins, create a supra-physiological hormonal environment, and promotes the growth of follicles, that under natural conditions would become atretic and regress (Setti et al., 2011).

The syndrome is characterized by cystic enlargement of the ovaries and a fluid shift from the intravascular to the third space due to increased capillary permeability and ovarian neoangiogenesis (Kumar et al., 2011). The incidence of moderate OHSS is estimated to be between 3\% and $6 \%$, and it has been recognized in two forms: the early form of OHSS, (within days after the ovulation triggering injection of hCG) although caused by hCG, it is related to an exaggerated ovarian response to COS, whereas the late form (10 days after hCG) (Abramov et al., 1999), is mainly related to the secretion of placental hCG. Those cases which constitute an early form followed by pregnancy are serious and long lasting (Golan et al., 1989).

Ovarian hyperstimulation syndrome results from an increase in vascular permeability. The hCG used to trigger oocyte maturation appears to play an integral part in the etiology of the condition and indeed, subsequent trophoblast-derived hCG dramatically worsens and prolongs the symptoms of severe OHSS (Whelan and Vlahos 2000; Aboulghar, 2009). Therefore, an important OHSS risk-reducing strategy has been to cancel the embryo transfer and freeze-all the embryos (Evans et al., 2014).

The cryopreservation of all embryos can prevent pregnancy-induced late OHSS; however, it cannot prevent early OHSS if hCG is used to trigger oocyte maturation (Endo et al., 2002)

The use of gonadotropin-releasing hormone agonist $(\mathrm{GnRHa})$ as a trigger for final oocyte maturation in antagonist in vitro fertilization (IVF) cycles has been proposed as a method for preventing ovarian OHSS (Cerrillo et al., 2011; Humaidan et al., 2011). From a clinical point of view, the most significant benefit of GnRHa trigger is its ability to induce a quick and reversible luteolysis and thus reduce the risk of OHSS development. Recently, it was demonstrated that gonadotropin and steroid levels differ significantly during the luteal phase between patients triggered for final oocyte maturation with a GnRHa or with hCG (Fatemi et al., 2013).

To date, there is no consensus in the literature on the use of GnRH to prevent the development of OHSS. Moreover, not much is known about clinical outcomes when $\mathrm{GnRH}$ agonist or hCG are used to trigger ovulation in freeze-all IVF cycles. Therefore, the goal for the present study was to compare the use of either GnRHa or hCG to trigger the final follicular maturation in freeze-all programs. We also aimed to compare the clinical outcomes when fresh versus freeze-thawed embryo transfers were performed in cycles with a high number of retrieved oocytes.

\section{MATERIALS AND METHODS}

\section{Experimental Design}

This is case-control study analyzing potential OHSS patients ( $>20$ retrieved MII) undergoing freeze-all cycles. The cycles were split into those receiving the GnRHa $(n=74)$ or the hCG $(n=49)$ trigger. The protocols were compared with respect to the clinical outcomes. We also compared the clinical outcomes of cycles in which hCG trigger was performed when the fresh embryo transfer protocol $(n=153)$ or (ii) the freeze-all protocol $(n=123)$ was performed.

The clinical parameters evaluated were: (i) pregnancy rate, (ii) single pregnancy rate, (iii) multiple pregnancy rate, (iv) implantation rate, (v) miscarriage rate and (vi) cumulative pregnancy rate.

The pregnancy test was performed 12 days after embryo transfer. All women with a positive test were submitted to a transvaginal ultrasound scan 2 weeks after the positive test. A clinical pregnancy was diagnosed when the 
fetal heartbeat was detected. Pregnancy rates were calculated per transfer. Miscarriage was defined as pregnancy loss before 20 weeks.

A written informed consent, in which patients agreed to share the outcomes of their cycles for research purposes was obtained, and the study was approved by the local institutional review board.

\section{Controlled Ovarian Stimulation and Oocyte Retrieval}

Controlled ovarian stimulation was achieved by using recombinant FSH (Gonal-F; Serono, Geneva, Switzerland), at a daily dose, starting on day three of the cycle. Pituitary blockage was performed using a GnRH antagonist (Cetrotide, Serono, Geneva, Switzerland), starting when at least one follicle $\geq 14 \mathrm{~mm}$ was visualized.

Follicular growth was monitored using transvaginal ultrasound examination starting on day four of gonadotropin administration. Recombinant hCG (Ovidrel, Serono, Geneva, Switzerland) or GnRHa (Leuprolide acetate, Lupron; TAP Pharmaceuticals, Lake Forest, USA) was administered to trigger the final follicular maturation. The oocytes were collected 35 hours after follicular maturation trigger through transvaginal ultrasound ovum pick-up.

\section{Preparation of oocytes and Morphology assessment}

Retrieved oocytes were maintained in culture medium (Global ${ }^{\circledR}$ for Fertilization, LifeGlobal, Connecticut, USA) supplemented with 10\% Human Synthetic Albumin (HSA, Irvine Scientific, Santa Ana, USA), covered with mineral oil (Ovoil $^{\mathrm{TM}}$ - Vitrolife, Kungsbacka, Sweden) at $37^{\circ} \mathrm{C}$ and $6 \%$ $\mathrm{CO} 2$ for 5 hours. Surrounding cumulus cells were removed with exposure to a HEPES buffered-medium containing hyaluronidase (80 IU/mL, Irvine Scientific, Santa Ana, USA). The remaining cumulus cells were then mechanically removed by gentle pipetting with a hand-drawn Pasteur pipette (Humagen Fertility Diagnostics, Charlottesville, Virginia, USA). The oocytes were checked for oocyte maturation and those which have released the first polar body (metaphase II oocytes - MII) were considered mature and used for ICSI.

\section{Intracytoplasmic Sperm Injection}

Intracytoplasmic Sperm Injection was performed on all MII oocytes using the technique described by Palermo et al. (1992). The oocytes were individually placed in 4- $\mu \mathrm{L}$ droplets of buffered medium (Global $®$ w/HEPES, LifeGlobal, Connecticut, USA), and sperm was placed in a central $4-\mu \mathrm{L}$ droplet of polyvinylpyrrolidone solution (PVP, Irvine Scientific, Santa Ana, USA) in a $50 \times 40-\mathrm{mm}$ glass culture dish (WillCo-dish $®$, New Jersey, USA) covered with warm mineral oil (Ovoil ${ }^{\mathrm{TM}}$, Vitrolife, Kungsbacka, Sweden), on a heated stage $\left(37.0 \pm 0.5^{\circ} \mathrm{C}\right)$ of an inverted microscope.

\section{Assessment of Fertilization and Embryo Quality}

After the ICSI procedure, the presumptive embryos were individually maintained in a $50-\mu \mathrm{L}$ drop of culture medium (Global ${ }^{\circledR}$, LifeGlobal, Connecticut, USA) supplemented with $10 \%$ human serum albumin (HAS) and covered with mineral oil in a humidified atmosphere with $6 \%$ $\mathrm{CO}_{2}$ at $37^{\circ} \mathrm{C}$ until transfer, which occurred on the fifty day of development.

Approximately $18 \mathrm{~h}$ after ICSI, fertilization was confirmed by the presence of two pronuclei and the extrusion of the second polar body. Subsequently, embryos were transferred to new drops of culture medium to be individually cultured for 48 hours. The quality of the embryos was evaluated under an inverted microscope.

\section{Embryo freezing or embryo transfer}

For freeze-all cycles, on day three, embryos were vitrified and cryo-stored. Both vitrification and the warming procedure were performed using the Cryotop method, described elsewhere (Quaas et al., 2013). On day five, for both fresh and freeze-all protocols, one or two embryos were transferred.

\section{Statistical analyses}

The cycle characteristics, clinical outcomes and laboratory outcomes were compared between the groups using Chi squared and student t-test for categorical and continuous variables, respectively. Continuous variables are expressed as the mean \pm the standard deviation, and percentages were used for categorical variables

All results considered $5 \%$ to be significant at the critical level $(P<0.05)$. Data analysis was carried out using the Minitab (version 14) Statistical Program.

\section{RESULTS}

When demographic variables, stimulation characteristics and response to COS were compared between cycles in which the trigger was performed by hCG or GnRHa, a decreased serum estradiol level, a decreased number of retrieved oocytes, an increased MII retrieved rate, and decreased fertilization rate were observed in the hCG group. No significant difference was noted for the other evaluated parameters (Table 1).

No significant difference was observed in the clinical outcomes when the trigger was per-

Table 1: Characteristics from freeze-all cycles when the trigger was performed by using hCG or GnRH agonist

\begin{tabular}{|l|c|c|c|}
\hline \multicolumn{1}{|c|}{ Cycles' Characteristics } & hCG & GnRH agonist \\
\hline Number of cycles & 49 & 74 \\
\hline Number of patients & 45 & $31.9 \pm 3.6$ & 0.887 \\
\hline Patient's age & $31.8 \pm 3.8$ & $5328 \pm 3036$ & 0.006 \\
\hline Estradiol level (E2) on trigger day & $4039 \pm 2112$ & $2067 \pm 481$ & 0.101 \\
\hline Total dose of FSH for COS & $2259 \pm 714$ & $41.3 \pm 17.9$ & 0.015 \\
\hline Aspirated follicles & $34.5 \pm 11,7$ & $30.8 \pm 11.3$ & $<0.001$ \\
\hline Retrieved oocytes & $25.3 \pm 9,6$ & $74.5 \%$ & 0.59 \\
\hline Retrieved oocytes rate & $73.4 \%$ & $22.0 \pm 8.1$ & 0.123 \\
\hline MII number & $19.6 \pm 7.8$ & $71.3 \%$ & $<0.001$ \\
\hline MII rate & $77.2 \%$ & $84.0 \%$ & 0.011 \\
\hline Normal fertilization rate & $79.3 \%$ & $9.9 \pm 4.9$ & 0.422 \\
\hline Number of cryopreserved embryos & $9.2 \pm 4.5$ & \multicolumn{2}{|c|}{} \\
\hline
\end{tabular}


formed by either hCG or GnRHa (Table 2).

When fresh cycles were compared with frozen-thawed cycles, the estradiol serum level on the trigger day and the number of cryopreserved embryos were higher in the frozen-thawed cycles. The other demographic variables, stimulation characteristics and response to COS variables did not differ between the groups (Table 3 ).

The clinical pregnancy rate was higher among freeze-all cycles, as well as the implantation and cumulative pregnancy rate, when compared with fresh embryo transfer cycles (Table 4).

\section{Table 2: Clinical outcomes from freeze-all cycles when the trigger was performed by using hCG or GnRH agonist}

\begin{tabular}{|l|c|c|c|}
\hline Cycle outcomes & hCG & $\begin{array}{c}\text { GnRH } \\
\text { agonist }\end{array}$ & $P$ \\
\hline Number of cycles & 49 & 74 & \\
\hline Clinical pregnancy rate & $44.8 \%$ & $50.0 \%$ & 0.483 \\
\hline Single pregnancy rate & $72.7 \%$ & $75.6 \%$ & 0.856 \\
\hline Twin pregnancy rate & $22.7 \%$ & $24.3 \%$ & 0.585 \\
\hline Triplet pregnancy rate & $4.5 \%$ & 0 & 0.935 \\
\hline Miscarriage rate & $29.7 \%$ & $14.6 \%$ & 0.164 \\
\hline Implantation rate & $39.0 \%$ & $37.1 \%$ & 0.885 \\
\hline Cumulative pregnancy rate & $53.0 \%$ & $59.5 \%$ & 0.483 \\
\hline
\end{tabular}

\section{DISCUSSION}

GnRHa can be used as an alternative trigger to hCG in cycles that have been suppressed with a GnRH antagonist. Prior studies of GnRHa triggering have reported complete prevention of severe early OHSS and good pregnancy rates following fresh embryo transfer even in high-risk patients (Humaidan 2009; Humaidan et al., 2009; Radesic \& Tremellen, 2011). Nevertheless, in a very elegant previous study (Iliodromiti et al., 2013) it was reported that in women undergoing ovarian stimulation and who develop an excessive ovarian response, the use of a GnRHa trigger combined can provide the opportunity to proceed to fresh embryo transfer with adequate clinical pregnancy rates. However, this procedure will not completely eliminate the risk of OHSS; therefore, in cases of extreme ovarian response, the GnRHa trigger followed by a freeze-all policy to completely avoid OHSS is recommended.

For the present study, the used of GnRHa trigger was compared with the hCG trigger in freeze-all protocols, and no differences were found for any evaluated parameter, with exception of fertilization and number of MII - which was increased and the MII retrieved rate, which was decreased in the GnRHa group.

The lower MII retrieved rate observed when GnRHa was used may be explained by the fact that differences exist between the GnRHa-induced surge and that of the natural cycle or the traditional hCG trigger. The LH surge of the natural cycle is characterized by three phases, with a total duration of $48 \mathrm{~h}$ (Hoff et al., 1983), whereas the GnRHa-induced surge of gonadotropins consists of two

\section{Table 3: Characteristics from OHSS cycles when fresh embryos were transferred or all of the embryos were} cryopreserved

\begin{tabular}{|l|c|c|c|}
\hline Cycles' Characteristics & Freeze-all & Fresh embryo transfer & $P$ \\
\hline Number of cycles & 123 & 153 & 141 \\
\hline Number of patients & 114 & $33.4 \pm 2.9$ & 0.431 \\
\hline Patient's age & $32.6 \pm 2,8$ & $3326 \pm 1657$ & 0.003 \\
\hline Estradiol level (E2) on trigger day & $4543 \pm 2232$ & $2298 \pm 756$ & 0.456 \\
\hline Total dose of FSH for COS & $2147 \pm 606$ & $37.9 \pm 6.9$ & 0.645 \\
\hline Aspirated follicles & $38.3 \pm 14,7$ & $27.4 \pm 11.2$ & 0.352 \\
\hline Retrieved oocytes & $28.2 \pm 10,9$ & $73.1 \%$ & 0.652 \\
\hline Retrieved oocytes rate & $73.5 \%$ & $20.4 \pm 6.7$ & 0.546 \\
\hline MII number & $20.8 \pm 8,0$ & $74.5 \%$ & 0.336 \\
\hline MII rate & $73.8 \%$ & $77.7 \%$ & 0.451 \\
\hline Normal fertilization rate & $80.7 \%$ & $5.2 \pm 3.1$ & $<0.0001$ \\
\hline Number of cryopreserved embryos & $9.3 \pm 4.6$ & & 2 \\
\hline
\end{tabular}

Table 4: Clinical outcomes from OHSS when fresh embryos were transferred or all of the embryos were cryopreserved

\begin{tabular}{|l|c|c|c|}
\hline Cycles' outcomes & Freeze-all & Fresh embryo transfer & $P$ \\
\hline Number of cycles & 123 & 153 & 0.004 \\
\hline Clinical pregnancy rate & $47.9 \%$ & $61.1 \%$ & 0.589 \\
\hline Single pregnancy rate & $74.5 \%$ & $31.7 \%$ & 0.384 \\
\hline Twin pregnancy rate & $23.7 \%$ & 0 & 0.754 \\
\hline Triplet pregnancy rate & $1.7 \%$ & $15.6 \%$ & 0.789 \\
\hline Miscarriage rate & $15.8 \%$ & $24.8 \%$ & 0.028 \\
\hline Implantation rate & $38.6 \%$ & - & \\
\hline Cumulative pregnancy rate & $62.3 \%$ & $55.7 \%$ & 0.049 \\
\hline Estimated cumulative pregnancy rate & $68.4 \%$ & & \\
\hline
\end{tabular}


phases only, with a duration of 24-36 h (Itskovitz et al., 1991). This leads to a significantly reduced total amount of gonadotropins being released from the pituitary when GnRHa is used. Conversely, the traditional hCG trigger continues to stimulate ovarian steroid hormone production for up to 5 days. For the GnRHa trigger, these differences may have an effect on oocyte maturation. The higher fertilization rate observed in this group may also be due to the "more physiological" environment for oocyte development, that is created when the GnRHa triggers follicular maturation.

When fresh and freeze-all protocols, following hCG trigger were compared, a significantly increased pregnancy rate was seen. In agreement with these findings, there is growing evidence in the literature suggesting that the supraphysiologic hormonal environment created by COS may decrease endometrial receptivity and embryo implantation (Devroey et al., 2004; Shapiro et al., 2014). In fact, it has been suggested that the freeze-all policy has emerged as an alternative to fresh embryo transfer to improve cycle outcomes (Roque et al., 2013; Roque et al., 2015).

In our study, the whole cohort of embryos were cryopreserved, and the embryo transfer was performed later in a most receptive and well-prepared endometria. The potential advantage of this method is that it provides a more physiologic hormonal milieu in which embryo transfers take place (Barnhart, 2014).

Barnhart et al. (2014) reported that ovarian stimulation may have unintended consequences. As many aspects of IVF have become optimized, other aspects, such as possible alterations in endometrial development, early embryo development, implantation, early placentation, and OHSS risk have become the focus of modifiable factors that may further enhance safety and success.

When comparing the cycles' characteristics of freezeall and fresh embryo transfer cycles, as expected, the estradiol serum level on the trigger day and the number of cryopreserved embryos were higher in the frozen-thawed cycles. Indeed the estradiol level is one of the main parameters in the decision to freeze the whole cohort of embryos in women undergoing ovarian stimulation and who develop an excessive ovarian response.

In conclusion, our findings suggest that the GnRHa trigger may be a good alternative to prevent OHSS in patients presenting an extreme ovarian response to COS, leading to similar clinical outcomes when compared with the traditional hCG trigger. Moreover, our findings demonstrated that the strategy of freezing all embryos not only decreases the risk of OHSS but it also leads to a better pregnancy rate.

\section{CONFLICT OF INTERESTS}

No conflict of interest have been declared.

\section{Corresponding author:}

Daniela Paes de Almeida Ferreira Braga

Fertility - Medical Group

Sao Paulo/SP - Brazil

E-mail: dbraga@fertility.com.br

\section{REFERENCES}

Aboulghar M. Symposium: Update on prediction and management of OHSS. Prevention of OHSS. Reprod Biomed Online. 2009; 19: 33-42.

Abramov Y, Elchalal U, Schenker J G. Severe OHSS: An 'epidemic' of severe OHSS: a price we have to pay? Hum Reprod. (1999); 14: 2181-3.

Barnhart KT. Introduction: are we ready to elim- inate the transfer of fresh embryos in in vitro fertilization? Fertil Steril. 2014; 102:1-2.

Cerrillo M, Pacheco A, Rodriguez S, Gomez R, Delgado F, Pellicer A, Garcia-Velasco J A. Effect of GnRH agonist and hCG treatment on VEGF, angiopoietin-2, and VE-cadherin: trying to explain the link to ovarian hyperstimulation syndrome. Fertil Steril. 2011; 95: 2517-9.

Devroey P, Bourgain C, Macklon NS, Fauser BC. Reproductive biology and IVF: ovarian stimulation and endometrial receptivity. Trends Endocrinol Metab. 2004;15:84-90.

Endo T, Honnma H, Hayashi T, Chida M, Yamazaki K, Kitajima Y, Azumaguchi A, Kamiya H, Kudo R. Continuation of GnRH agonist administration for 1 week, after hCG injection, prevents ovarian hyperstimulation syndrome following elective cryopreservation of all pronucleate embryos. Hum Reprod. 2002; 17: 2548-51.

Evans J, Hannan N J, Edgell T A, Vollenhoven B J, Lutjen $P$ J, Osianlis T, Salamonsen L A, Rombauts L J. Fresh versus frozen embryo transfer: backing clinical decisions with scientific and clinical evidence. Hum Reprod Update. 2014; 20: 808-21.

Fatemi H M, Polyzos N P, van Vaerenbergh I, Bourgain C, Blockeel C, Alsbjerg B, Papanikolaou E G, Humaidan P. Early luteal phase endocrine profile is affected by the mode of triggering final oocyte maturation and the luteal phase support used in recombinant follicle-stimulating hormone-gonadotropin-releasing hormone antagonist in vitro fertilization cycles. Fertil Steril. 2013; 100: 742-7.

Golan A, Ron-el R, Herman A, Soffer Y, Weinraub Z, Caspi E. Ovarian hyperstimulation syndrome: an update review. Obstet Gynecol Surv. 1989; 44: 430-40.

Hoff JD, Quigley ME, Yen SS. Hormonal dynamics at midcycle: a reevaluation. J Clin Endocrinol Metab. 1983; 57:792-6.

Humaidan P. Luteal phase rescue in high-risk OHSS patients by GnRHa triggering in combination with low-dose HCG: a pilot study. Reprod Biomed Online. 2009; 18: 630-4.

Humaidan P, Kol S, Papanikolaou EG; Copenhagen GnRH Agonist Triggering Workshop Group; GnRH agonist for triggering of final oocyte maturation: time for a change of practice? Hum Reprod Update. 2011; 17: 510-24.

Humaidan P, Papanikolaou EG, Tarlatzis BC. GnRHa to trigger final oocyte maturation: a time to reconsider. Hum Reprod. 2009; 24: 2389-94.

Iliodromiti S, Blockeel C, Tremellen KP, Fleming R, Tournaye $H$, Humaidan $P$, Nelson $S M$. Consistent high clinical pregnancy rates and low ovarian hyperstimulation syndrome rates in high-risk patients after $\mathrm{GnRH}$ agonist triggering and modified luteal support: a retrospective multicentre study. Hum Reprod. 2003; 28: 2529-36.

Itskovitz J, Boldes R, Levron J, Erlik Y, Kahana L, Brandes JM. Induction of preovulatory luteinizing hormone surge and prevention of ovarian hyperstimulation syndrome by gonadotropin-releasing hormone agonist. Fertil Steril. 1991; 56: 213-20.

Kumar P, Sait SF, Sharma A, Kumar M. Ovarian hyperstimulation syndrome. J Hum Reprod Sci. 2011;4: 70-5. 
Palermo G, Joris H, Devroey P, Van Steirteghem AC. Pregnancies after intracytoplasmic injection of single spermatozoon into an oocyte. Lancet. 1992; 340: 17-8.

Quaas AM, Melamed A, Chung K, Bendikson KA, Paulson RJ. Egg banking in the United States: current status of commercially available cryopreserved oocytes. Fertil Steril 2013; 99: 827-31.

Radesic B, Tremellen K. Oocyte maturation employing a $\mathrm{GnRH}$ agonist in combination with low-dose hCG luteal rescue minimizes the severity of ovarian hyperstimulation syndrome while maintaining excellent pregnancy rates. Hum Reprod. 2011; 26: 3437-42.

Roque M, Lattes K, Serra S, Sola I, Geber S, Carreras R, Checa MA. Fresh embryo transfer versus frozen embryo transfer in in vitro fertilization cycles: a systematic re- view and meta-analysis. Fertil Steril. 2013; 99:156-62.

Roque M, Valle M, Guimaraes F, Sampaio M, Geber S. Freeze-all policy: fresh vs. frozen-thawed embryo transfer. Fertil Steril. 2015; 103: 1190-3.

Setti AS, Figueira RC, Braga DP, Colturato SS, Iaconelli Jr A, Borges Jr. E. Relationship between oocyte abnormal morphology and intracytoplasmic sperm injection outcomes: a meta-analysis. Eur J Obstet Gynecol Reprod Biol. 2011; 159: 364-70.

Shapiro BS, Daneshmand ST, Garner FC, Aguirre M, Hudson C. Clinical rationale for cryopreservation of entire embryo cohorts in lieu of fresh transfer. Fertil Steril. 2014; 102: 3-9.

Whelan JG 3rd, Vlahos NF. The ovarian hyperstimulation syndrome. Fertil Steril. 2000; 73: 883-96. 